\title{
Chalcatzingo, México: donde las piedras hablan
}

Chalcatzingo, Mexico: Where Stones Talk

\author{
Juan Francisco Illescas Salinas \\ Universidad Autónoma Metropolitana, \\ Unidad Azcapotzalco (UAM-A), México \\ jfis@azc.uam.mx
}

\section{Gilberto Buitrago Sandoval}

Restaurador independiente, Colombia

buitragosandoval@hotmail.com

\section{Resumen}

\begin{abstract}
El presente INFORME versa sobre los alcances del Proyecto de Conservación y Restauración de los Elementos Arqueológicos de Chalcatzingo (Coordinación Nacional de Conservación del Patrimonio Cultural [CNCPC] y Centro INAH-Morelos, ambas dependencias del Instituto Nacional de Antropología e Historia [INAH, México]), que a lo largo de cuatro años (2011-2014) ha desarrollado labores de investigación e intervención en los famosos bajorrelieves en piedra que muestran la impronta de la cultura olmeca en este sitio arqueológico del estado de Morelos, México. Se presentan los resultados correspondientes al estudio material de los soportes rocosos, el cual incluye una revisión bibliográfica del contexto geológico del sitio, una caracterización petrográfica de muestras procedentes de algunos bajorrelieves y una aproximación a su estado de deterioro. Asimismo, se describen cronológicamente las intervenciones realizadas in situ, las cuales se enfocaron en la estabilización, conservación preventiva y optimización del entorno de dichos monumentos arqueológicos.
\end{abstract}

\section{Palabras clave}

conservación; bajorrelieve; Chalcatzingo, México; caracterización petrográfica; deterioro; conservación preventiva

\section{Abstract}

This INVESTIGATION REPORT addresses the achievements of the Conservation and Restoration of Archaeological Features Project from Chalcatzingo (National Coordination of Conservation and Cultural Heritage [CNCPC] and INAH-Centre Morelos, both depending from the National Institute of Anthropology and History [INAH, México]). During this four-year project (2011-2014) research and intervention was carried out of the famous stone bas-reliefs that show the imprints of 
the Olmec culture in this archaeological site in the state of Morelos, Mexico. This contribution presents the results of the material study of the rock support --which include a bibliographic review of the geological context of the site and a petrographic characterization of the samples from some of the bas-reliefs-. After providing an assessment of the condition of deterioration of the carvings, it also describes -in chronological order - the interventions that were carried out in situ that focused on stabilization, preventive conservation and improvement of the environment of the archaeological monuments.

\section{Key words}

conservation; bas-relief; Chalcatzingo; Mexico; petrographic characterization; decay; preventive conservation

\section{Introducción}

$\longrightarrow$ halcatzingo, localizado en la parte oriental del estado de Morelos, es un yacimiento arqueológico que ha sido reconocido como una impronta del arte monumental y de la iconografía del estilo olmeca en el centro de México (Grove y Angulo 1987:132-158; Grove 1999:255, 2008:1-7, 2010:1-8; Córdova Tello y Meza Rodríguez 2007: 60-65). Se afirma que el asentamiento se fundó alrededor del siglo XV a. C. y que su florecimiento, durante el cual se perfeccionó el arte monumental en piedra, data de los siglos VII y $\vee$ a. C., que corresponde al periodo Preclásico Medio y Tardío de la cronología mesoamericana, época en la que se constituyó como un centro político-religioso de gran importancia regional (Grove 1999:288). En la actualidad sobrevive una serie de bajorrelieves tallados en grandes y me- dianos fragmentos de roca procedentes, supuestamente, del propio cerro Chalcatzingo (Figura 1).

Estos monumentos arqueológicos, que se han convertido en un referente importante del lugar, hacen de Chalcatzingo un sitio de gran relevancia arqueológica (Figura 2) (Grove 1999: 255-295, 2008: 1-7; 2010: 1-8). En virtud de tal valoración, en fechas recientes sus bajorrelieves han sido objeto de investigación e intervención en materia de conservación, iniciativas que constituyen el tema de este INFORME.

\section{Antecedentes}

La arqueóloga Eulalia Guzmán descubrió, oficialmente en 1934, los monumentos arqueológicos que aquí nos ocupan cuando, después de una tormenta, los habitantes del lugar señalaron la presencia de cuatro piedras con bajorrelieves; siendo la mayor

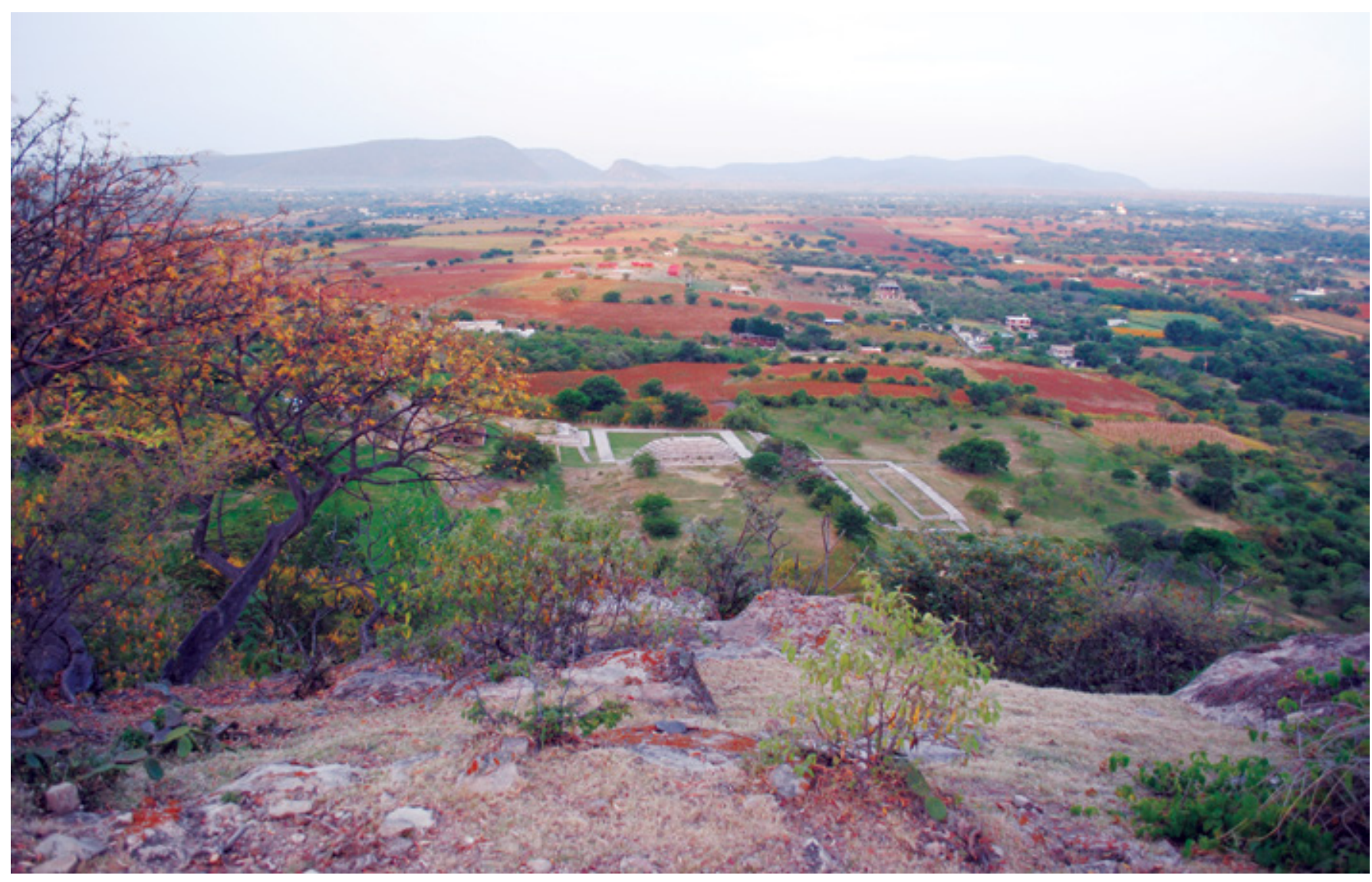

FIGURA 1. Vista general de la Zona Arqueológica de Chalcatzingo, Morelos, México (Cortesía: Proyecto de Conservación y Restauración de los Elementos Arqueológicos de Chalcatzingo, CNCPC-INAH). 


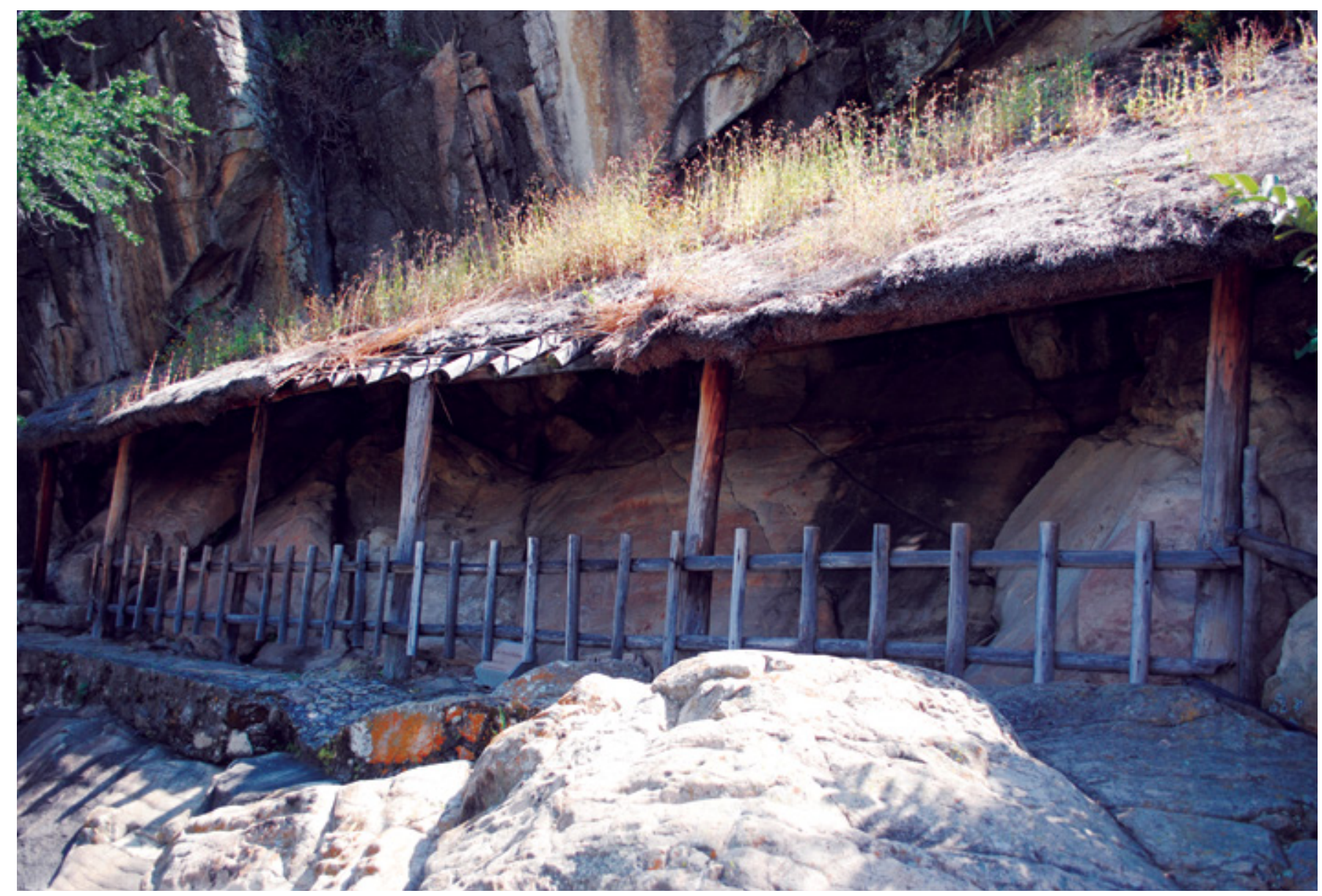

FIGURA 2. Bajorrelieve El rey de la Zona Arqueológica de Chalcatzingo, Morelos, México (Cortesía: Proyecto de Conservación y Restauración de los Elementos Arqueológicos de Chalcatzingo, CNCPC-INAH).

de ellas, el Monumento 1, al que se denominó El rey (Grove 1987b:2). Con el transcurrir del tiempo se registraron más monumentos tallados en Chalcatzingo. Un primer catálogo pormenorizado de éstos se compiló en 1972, cuando se le encargó a los arqueólogos David Grove, Raúl Arana y Jorge Angulo la dirección del Proyecto Arqueológico Chalcatzingo (PAC), propuesta a la que se unieron la University of Illinois at Chicago (UIC), Estados Unidos de América (EUA) y el Instituto Nacional de Antropología e Historia (INAH), México (Grove y Angulo 1987:132-158; Córdova Tello y Meza Rodríguez 2007: 60-65). La publicación más importante de este proyecto es, sin duda, Ancient Chalcatzingo (Grove 1987), compilación en la que diversos autores describen, analizan y clasifican los bajorrelieves, un corpus bibliográfico fundacional para la investigación de la iconografía, la cultura y el desarrollo de los olmecas en el centro de México.

Hoy en día la Zona Arqueológica de Chalcatzingo exhibe 41 bajorrelieves, todos ellos tesoros invaluables de nuestro patrimonio arqueológico, un legado escultórico que debe protegerse para el estudio y regocijo de generaciones futuras. Para ello, desde el año 2010, auspiciado por el PAC, que dirige el arqueólogo Mario Córdova Tello, la Coordinación Nacional de Conservación del Patrimonio Cultural (CNCPC) y el Centro INAH-Morelos, ambas del INAH, han sumado esfuerzos en pos de la preservación del legado escultórico de este sitio. Las labores, que han tenido lugar principalmente en cuatro temporadas de trabajo de campo (CNCPC-INAH y Centro INAH-Morelos
2010, 2011, 2012, 2013), se han caracterizado por una orientación básicamente preventiva, cuya complejidad ha aumentado concomitantemente al desarrollo del Proyecto de Conservación y Restauración de los Elementos Arqueológicos de Chalcatzingo, cuya planeación elaboró el restaurador Luis Rogelio Rivero Chong (CNCPC-INAH). Efectivamente, a la atención inicial de necesidades básicas de mantenimiento, preservación y conservación directa de los elementos arqueológicos expuestos a las inclemencias de la intemperie - que prácticamente no habían sido intervenidos por restauradores profesionales desde su descubrimiento- se ha sumado un estudio material sobre los bajorrelieves. Las próximas líneas informan de los alcances obtenidos en cada uno de estos rubros. 
Estudio material de los bajorrelieves

El estudio material de los monumentos de Chalcatzingo dio principio con un acercamiento bibliográfico al contexto geológico regional, tema fundamental para entender la naturaleza de las rocas que sirvieron para la elaboración de los bajorrelieves, el cual se complementó tanto con un primer análisis de materiales constitutivos como con una aproximación inicial a su deterioro.

\section{Contexto geológico regional}

La Zona Arqueológica de Chalcatzingo, ubicada en el área meridional de las tierras altas del centro de México, concretamente en el centro del valle de Morelos, forma parte de un paisaje regional que representa uno de los pocos vestigios de la Franja Volcánica Transmexicana ancestral (Mioceno inferior, $\approx 21 \mathrm{Ma}$ ) (Mori 2007:11-15). Ubicadas en el sector oriental de un arco magmático continental (relacionado con la subducción de las placas oceánicas de Cocos y Rivera, por debajo de la placa de América del Norte a lo largo de la trinchera mesoamericana), las tres protuberancias que emergen drásticamente del valle y circundan el sitio son afloramientos constituidos por restos de cuellos volcánicos, que formaron, por una parte, un cuerpo grande conocido como cerro Chalcatzingo, y por otra dos pequeños, denominados cerro Delgado y cerro del Coyote (Mori 2007:11-15).

Expuestos a un clima cálido subhúmedo caracterizado por una temperatura promedio anual de más de $22{ }^{\circ} \mathrm{C}$ y una precipitación promedio anual que fluctúa entre 800 y 1000 mm (Werre Keeman y OrtizHernández 2000), los domos del cerro Chalcatzingo son formaciones geológicas resistentes a la erosión que permanecen como relictos de un cono volcánico desaparecido; y que por su origen están constituidos por rocas ígneas intrusivas hipoabisales, es decir, aquellas resultantes de la consolidación de magma volcánico en el interior de la litosfera en condiciones de baja presión, ${ }^{1}$ las cuales se consideran intermedias entre rocas plutónicas y volcánicas (Mori 2007:11-15). Si bien a lo largo de la evolución geológica de la región existen numerosas evidencias de diferentes regímenes tectónicos, estudios geofísicos reportan un lineamiento de dirección NE-SO (Mori 2007:11-15); de ahí que sus domos presenten una fracturación característica que domina el paisaje.

La información geológica hasta aquí vertida estableció el marco para emprender un análisis de materiales constitutivos basado en estudios macro y microscópicos de una serie de muestras provenientes de seis bajorrelieves: La creación del hombre, El altar olmeca, Estela del cazador, El puma, La danza de la fertilidad y El dador de agua, cuyos soportes presumiblemente corresponden a los domos del cerro Chalcatzingo.

\section{Análisis de materiales constitutivos}

El análisis de materiales constitutivos de los bajorrelieves de Chalcatzingo partió de una descripción macroscópica de las muestras, que reportó una estructura homogénea formada por algunos cristales observables a simple vista (fenocristales) englobados en una matriz de color beige. Éstos se presentaron con tonalidades distintivamente claras, correspondientes a plagioclasa y feldespato, así como con coloraciones oscuras, respectivas a minerales ferromagnesianos (piroxenos y anfíboles). En cuanto a la matriz, se registró que está formada por cristales visibles únicamente por el microscopio, es decir, con texturas afaníticas. Mediante dicha observación microscópica se identificó, asimismo, el color beige general de las rocas, atribuible a la silicifica-

\footnotetext{
1 Concretamente, los cuellos volcánicos de Chalcatzingo se formaron en el interior de conductos volcánicos en un ambiente de poca profundidad (Mori 2007).
}

ción ${ }^{2}$ y/o cloritización ${ }^{3}$ de su matriz. Respecto del tamaño relativo de grano, en todas las muestras de estudio se observaron grandes diferencias entre los fenocristales y los cristales de la matriz.

Con el fin de ahondar en la caracterización de las rocas se elaboraron láminas delgadas de las muestras referidas, las cuales se sometieron a observación en un microscopio óptico - técnica utilizada principalmente para reconocer los minerales presentes e identificar la textura (disposición de los elementos constitutivos de las rocas) - de polarización marca Carl Zeiss, modelo Axiolab Pol, adaptado con un analizador de imágenes KS 300 (Figura 3). Como resultado de este estudio se documentó que todas las muestras presentan textura holocristalina, esto es, que en su totalidad están compuestas por cristales y no poseen vidrio. En relación con los tamaños relativos de grano, todas las muestras poseen una textura porfídica, que consiste en algunos fenocristales relativamente grandes (de formación temprana) y megacristales englobados en una matriz formada por cristales no observables a simple vista. En lo tocante a la morfología de los cristales, en todos los casos se observaron texturas hipidiomórficas, que corresponden a un carácter intermedio en cuanto a la definición de caras y ángulos de los cristales.

La caracterización petrográfica se hizo con base en una nomenclatura de la naturaleza "volcánica" de las rocas derivada del análisis geológico. Las muestras de los bajorrelieves se caracterizaron como andesitas, a excepción del soporte pétreo del

\footnotetext{
2 Introducción y/o neoformación de sílice a partir de los minerales preexistentes en la roca, los cuales suelen eliminarse casi totalmente.

${ }^{3}$ Transformación de cualquier mineral ferromagnesiano en clorita, un filosilicato muy hidratado que comúnmente reemplaza a este tipo de minerales menos hidratados a bajas temperaturas, cuando hay disponibilidad de agua.
} 
bajorrelieve Estela del cazador, que, a partir de la abundancia de los minerales primarios, se identificó como basalto. Adicionalmente, con base en la abundancia de elementos químicos (diagrama TAS) encontrados en las muestras, se determinó que la $E s$ tela del cazador se elaboró en una roca básica (contenido de $\mathrm{SiO}_{2}$ entre $45 \%$ y $52 \%$ en peso), mientras que el resto se talló en rocas intermedias (contenido de $\mathrm{SiO}_{2}$ entre $52 \%$ y $63 \%$ en peso). Es de notar que en sus láminas delgadas también se observaron rasgos distintivos: fenocristales de plagioclasa y piroxeno (clino y ortopiroxeno) rodeados por una matriz formada por microlitos de plagioclasa con minerales opacos asociados y microcristales de piroxeno; cristales de plagioclasa poco alterados con típicas maclas y zonaciones; y fenocristales formando glomeropórfidos tanto de plagioclasa y piroxeno como de piroxenos. Dado que en las láminas delgadas caracterizadas, por una parte, existe un mayor contenido en plagioclasa que en piroxeno, y por otra, no se han observado anfíboles, se concluye que este monumento se elaboró en un basalto (anortita en plagioclasa $>50 \%$ ) toleítico (por su ausencia de cristales de olivino), que destaca por un estado "sano" o libre de alteraciones.

\section{Aproximación al deterioro}

La caracterización de las rocas mediante la observación a simple vista y el estudio de láminas delgadas en microscopio óptico también pusieron de manifiesto la presencia de algunos indicadores y productos de alteración que nos aproximan a comprender el deterioro de los bajorrelieves de Chalcatzingo.

Vale la pena señalar la presencia de productos de alteración derivados de origen, resultado de típicos procesos tardimagmáticos (i.e., incorporación de ciertos elementos químicos durante la cristalización del magma). En concreto, en las láminas delgadas de la mayoría de las muestras se observaron evidencias de procesos de
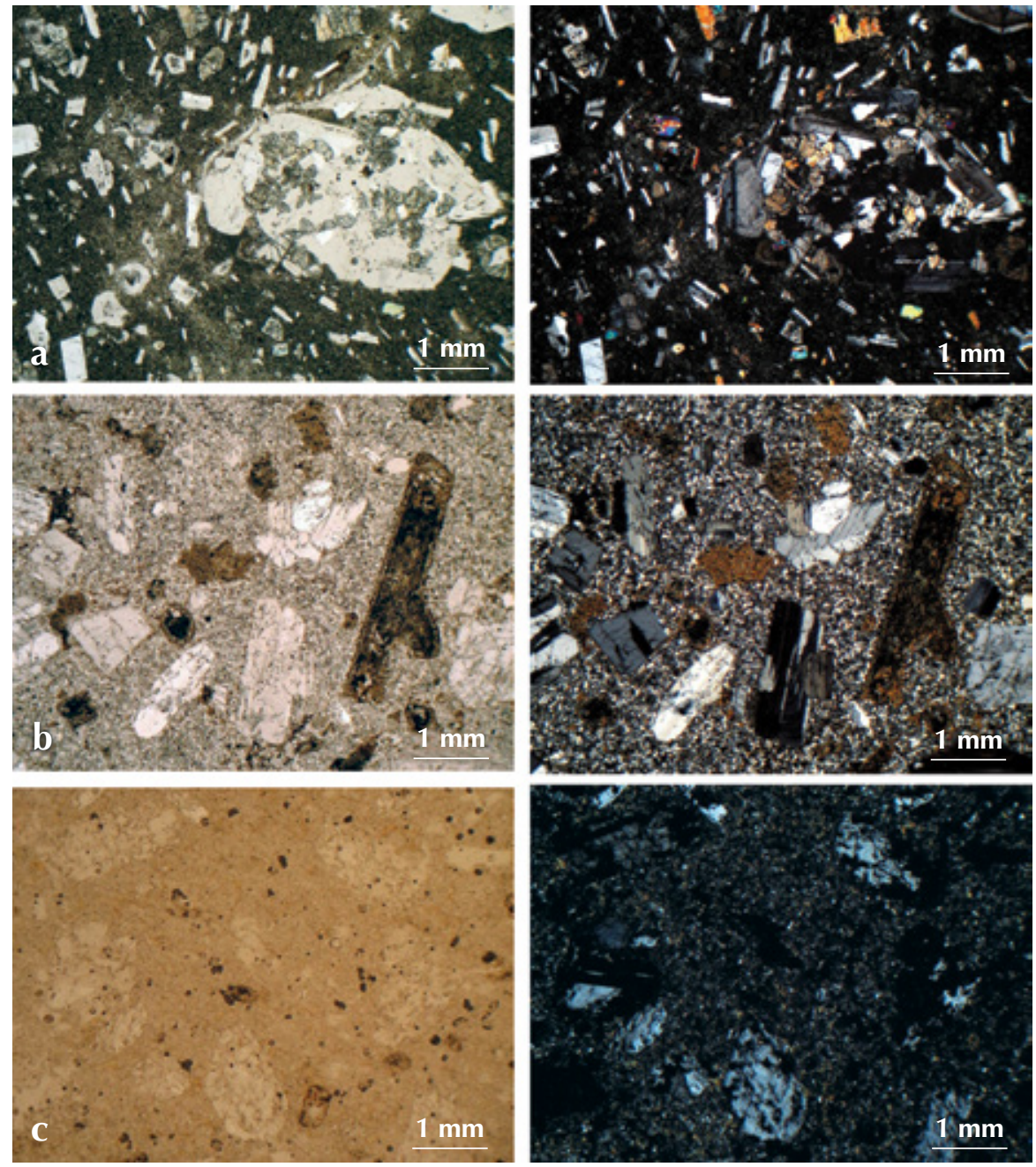

FIGURA 3. Imágenes de láminas delgadas correspondientes a muestras representativas de los bajorrelieves, obtenidas mediante microscopía óptica en condiciones de luz transmitida. En cada caso, se muestra a la izquierda una imagen obtenida con un nicol, y a la derecha la misma imagen con nicoles cruzados: a. Glomeropórfido formado por plagioclasa y piroxeno, de una muestra procedente del relieve Estela del cazador; b. Fenocristales de plagioclasa y de minerales ferromagnesianos rodeados por una matriz microcristalina, de una muestra procedente del relieve $\mathrm{El}$ altar olmeca; c. Fenocristales de feldespato rodeados por una matriz alterada, de una muestra procedente del relieve La danza de la fertilidad (Cortesía: Proyecto de Conservación y Restauración de los Elementos Arqueológicos de Chalcatzingo, CNCPC-INAH).

cloritización y silicificación, transformaciones que suelen ser consecuencia de la interacción entre los minerales formados en equilibrio con el magma y el agua y/o el aire.

Durante el estudio se detectaron otras alteraciones en las rocas, derivadas de la sinergia de varios factores, como la naturaleza química de las mismas, la fisiografía del entorno arqueológico y las condiciones medioambientales a las que han estado sometidas. En términos de morfología macroscópica se advirtieron tres tipos fundamentales de indicadores visuales de alteración:
- Pérdida de material constitutivo (con y sin formación de huecos), la cual puede ser consecuencia de acciones mecánicas o fisicoquímicas. Este tipo de alteración está relacionada con la acción conjunta del agua y el viento sobre las superficies pétreas. En el caso del entorno arqueológico específico, su zona central se encuentra atravesada por un barranco por el que circula agua de lluvia, lo que acentúa la acción del agua como agente de alteración. La gran cantidad de agua de lluvia que fluye mayoritariamente durante los meses de 
julio, agosto y septiembre genera el deslave de material arcilloso y ayuda a que bloques de roca de diferentes tamaños se transporten pendiente abajo.

- Modificaciones superficiales que afectan esencialmente al aspecto exterior de la roca.

- Rupturas (discontinuidades sin pérdida de material) y disyunciones (separaciones abiertas entre partes).

En la mayoría de las muestras de los bajorrelieves de Chalcatzingo se descubrió que el principal indicador de alteración corresponde al primer tipo, es decir, a la pérdida de material constitutivo, con la respectiva formación de oquedades, que se detectan como un estriado a manera de surcos en las zonas de menor cohesión. Sin embargo, también son evidentes indicadores de pérdida de material constitutivo sin formación de oquedades, derivados de tres procesos:

- Erosión, que provoca la reducción de relieve por acción de agentes atmosféricos de acción fisicoquímica.

- Disgregación, manifestada por el desprendimiento de gránulos o cristales derivado de la acción de los más mínimos estímulos mecánicos.

- Arenización, caracterizada por la disgregación y pérdida, generalmente inducida, de materia en forma de granos.

Adicionalmente, el análisis a simple vista detectó una serie de modificaciones superficiales de alteración, a seguir:

- Cromatización, manifestada a través de una variación de color.

- Presencia de manchas de pequeñas áreas, fundamentalmente de origen biológico.

- Formación de concreciones formadas por una cubierta exterior endurecida.
- Depósito superficial por acumulación de material de diversa naturaleza.

Derivado del estudio macro y microscópico se identificaron, asimismo, tres principales indicadores de alteración por ruptura:

- Fracturación, derivada de la acción de soluciones de continuidad sin desplazamiento relativo de las dos partes.

- Fisuración, manifestada en la formación de soluciones de continuidad con separación macroscópica de las dos partes.

- Fragmentación, que implica una pérdida de material.

A éstos se suman tres principales indicadores de alteración por disyunción:

- Placas o láminas extensas de varios milímetros de espesor.

- Escamas con forma y espesor irregular y dimensiones variables.

- Exfoliación, manifestada por un levantamiento seguido de la separación de una o más láminas paralelas entre sí.

Aparte de lo anterior, vale la pena subrayar que, al estar expuestos a la intemperie, los monumentos arqueológicos de Chalcatzingo han sido objeto de un importante biodeterioro. Efectivamente, factores como la Iluvia, el viento, el sol, el hielo y los contaminantes atmosféricos provocan un aumento de la superficie específica del material pétreo por formación de macro y microfisuras, así como rugosidades, lo cual favorece la colonización de organismo vivos, cuya presencia se asocia con procesos de alteración físicos y químicos: algunas especies de microorganismos son capaces de generar sustancias que alteran irreversiblemente la roca (García Murillo 1995).

Tanto en las muestras analizadas como en los propios bajorrelieves se hicieron patentes a escala macroscó- pica especies de líquenes -organismos pioneros en la colonización de las rocas-, musgos y plantas superiores (Figura 4), cada uno de los cuales contribuye de forma específica al biodeterioro del soporte pétreo.

Los líquenes, al poseer un crecimiento lento con pocos requerimientos ecológicos, son muy resistentes a condiciones extremas de temperatura y humedad, dañando no sólo estética y físicamente el sustrato pétreo, sino también químicamente, debido tanto a la condensación y retención de agua que generan sobre la superficie pétrea como a la secreción de ácidos orgánicos producidos por su metabolismo (MedinaGonzález y Schneider 1993:28-31). Por su parte, los musgos - que para crecer requieren un mínimo estrato de suelo para anclar sus raíces y la presencia continua de agua sobre una roca preferiblemente porosa (García Murillo 1995:56-60)— deterioran la roca, principalmente, a causa de tres mecanismos físicos y químicos: penetración en el sustrato de rizoides que siguen el sistema de vesículas; extracción de cationes debida a la acidez de sus raíces; y enriquecimiento en materia orgánica e inorgánica que, a su vez, propicia el desarrollo de plantas superiores. Estas últimas son las más destructivas, pues ocasionan alteraciones mecánicas, químicas y estéticas derivadas tanto de la presión ejercida por las raíces, seguida del crecimiento y el engrosamiento radial, como de la acción de los exudados de sus raíces, que contienen compuestos orgánicos e inorgánicos (García Murillo 1995:56-60).

\section{Intervenciones de conservación}

Las labores de campo del Proyecto de Conservación y Restauración de los Elementos Arqueológicos de Chalcatzingo, CNCPC-INAH, tuvieron lugar en cuatro temporadas consecutivas, de 2010 a 2013, cuyos alcances describimos a continuación. 

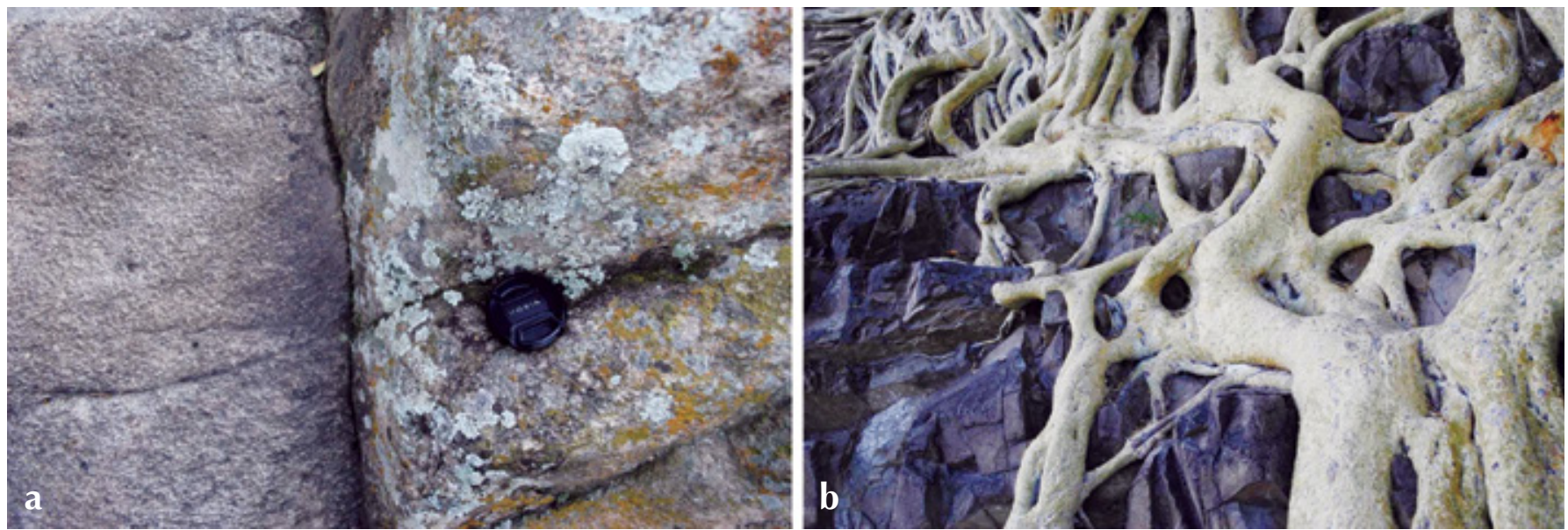

FIGURA 4. Fotografías de algunos organismos colonizadores de la roca del entorno del yacimiento arqueológico: a. Líquenes en forma de incrustaciones y manchas con coloraciones amarilla y verde clara, asociados a musgos en forma de talos verdes-grisáceos; b. Crecimiento de raíces de plantas superiores sobre el sustrato pétreo (Cortesía: Proyecto de Conservación y Restauración de los Elementos Arqueológicos de Chalcatzingo, CNCPC-INAH).

Primera temporada (junio-julio de 2010)

Al inicio de esta primera temporada de trabajo se realizó un levantamiento de prioridades de atención en el sitio que derivó en mandatos de documentación y diagnosis de relieves, de selección de casos de urgente estabilización y de ejecución de acciones de conservación preventiva.

La documentación privilegió la toma de fotografías como medida de registro y monitoreo, tarea que se ha mantenido a lo largo de todo el proyecto. Durante la diagnosis se reparó en que la mayoría de los relieves se encontraba afectada por exfoliación, disgregación, erosión y abrasión producidas por la intensa incidencia de vientos y lluvias estacionales. Se constató, además, que la alteración más notoria era la presencia de grandes manchas negras provocada por el crecimiento de microorganismos sobre el soporte rocoso. Asimismo, se advirtieron casos específicos de monumentos con problemáticas de estabilidad y de lectura que requerían atenderse de forma urgente: la Estela 21 o La reina, la Estela 27 o El cazador, el Monumento 25 o Altar circular; así como la serie conformada por los relieves siguientes: 60 Guía de planta de calabaza, 7 o Animal mítico sobre doble vírgula, 8 o Animal mítico cocodrilo de cola an- cha, 11 o Animal mítico sobre doble vírgula con doble gota de agua, 14 o Animal mítico sobre doble vírgula con lengua bífida, y 15 o Animal mítico con nube y gotas de lluvia. Todos ellos se intervinieron mediante procesos de limpieza en seco, unión de fragmentos con pernos y resina epóxica, consolidación por aplicación de resanes a base de polvo de piedra del lugar y cal apagada que, en proporción $2: 1$, se reintegraron cromáticamente al tono de la roca.

Con el fin de minimizar la acción de los agentes de intemperismo se puso en práctica un programa de elaboración de cubiertas de protección que inició con el reemplazo de aquellas con deficiencias de estabilidad en su desempeño. Un primer diseño de cubierta, prácticamente fabricado con material reciclado, correspondió al relieve denominado La reina, el cual buscó, con la mínima afectación a la apreciación del elemento y su entorno, proporcionarle protección contra la incidencia directa de la lluvia, la luz solar, los cambios de temperatura y la erosión eólica. La solución técnica partió de tender una estructura con tubos de metal pintados con anticorrosivo que emplea como tirantes unas tiras de zacate obtenido de los techos anteriores, a cuyo marco interior se lo dotó de aluminio para sostener dos láminas de policarbo- nato "ahumado" de $8 \mathrm{~mm}$ de espesor unidas por medio de remaches. Alrededor del monumento se dispuso un piso delgado de cemento, sobre el cual se depositó grava con el propósito de controlar la acumulación de tierra que propicia la colonización biológica (Figura 5).

Este diseño piloto funcionaría después en el sitio como modelo para la elaboración de otras techumbres de protección que obedecerían a los requerimientos planteados: ser sencillas, económicas, reutilizables y fáciles de armar-desarmar. Por último, debido a riesgos asociados a su entorno, se reubicaron dos estelas: La reina y El altar circular.

\section{Segunda temporada (marzo-junio} de 2011)

Las labores de esta temporada principiaron con una evaluación de las intervenciones realizadas un año antes. De manera general, se observó que los relieves intervenidos no presentaban nuevas alteraciones, salvo la acumulación de polvo superficial; en lo particular, se comprobó que las cubiertas erigidas durante la temporada anterior estaban cumpliendo con su función. Con estas bases se procedió a intervenir otra serie de relieves: los monumentos 2 o La danza de la fertilidad, 4 o Linaje de los gobernantes, 13 o El gobernador, 


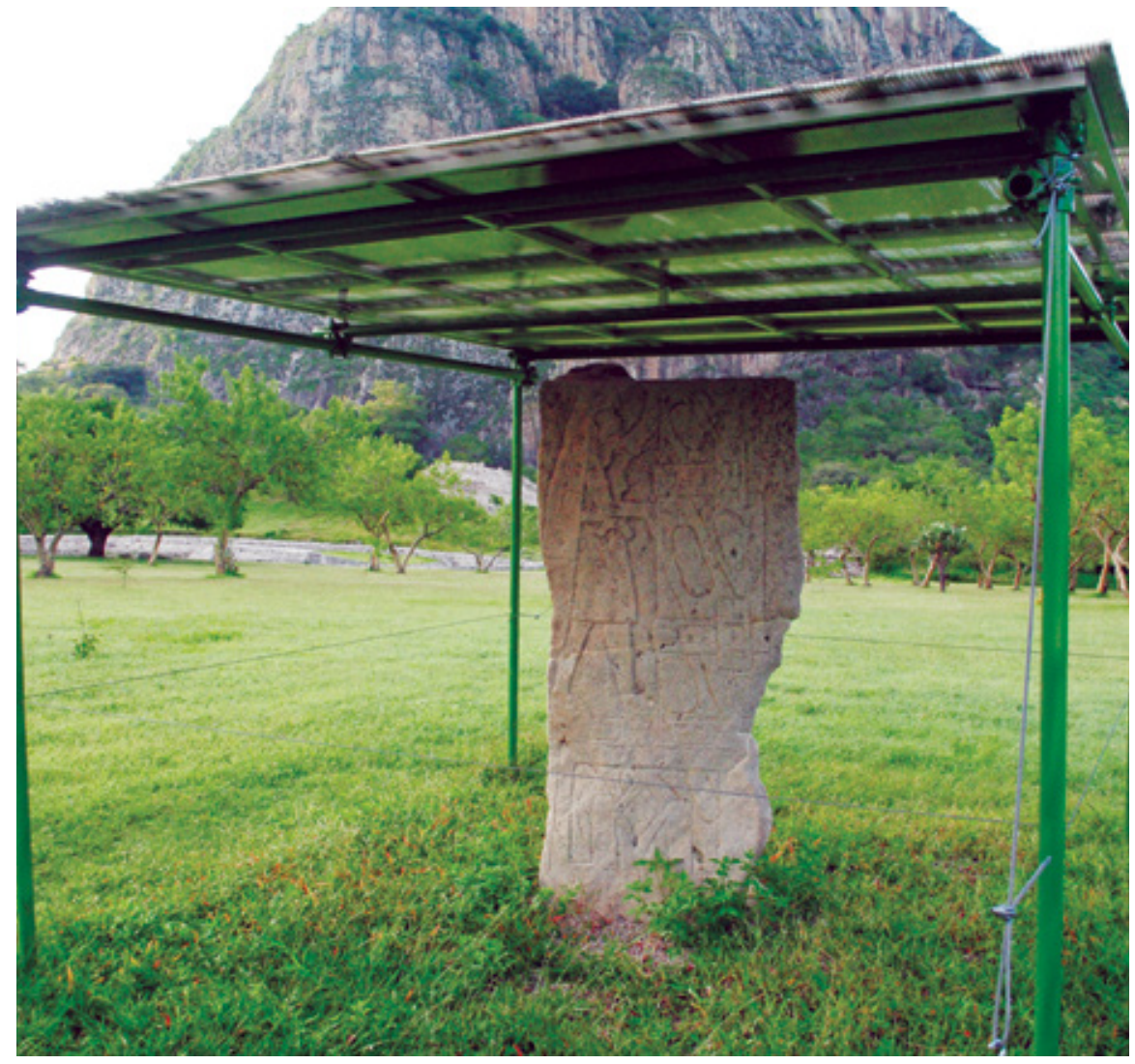

FIGURA 5. Estela 21 o La reina después de la intervención, Zona Arqueológica de Chalcatzingo, Morelos, México (Cortesía: Proyecto de Conservación y Restauración de los Elementos Arqueológicos de Chalcatzingo, CNCPC-INAH).

y 31 o Felino agazapado, acciones que concurrieron con la lógica, los materiales y los procedimientos ya empleados. Adicionalmente, se intervino en el entorno de los relieves mediante la creación de senderos de circulación que favorecieran el acceso óptimo y debidamente distante del observador, lo cual contribuyó a la protección de aquéllos. Otra de las medidas preventivas fundamentales, particularmente a lo largo del corredor donde se ubican los monumentos 3, 4, 5 y 31, fue la construcción de un muro de contención que en época de lluvias conduce el impacto de los pequeños aluviones generados por acarreo de material de depósito.

Las acciones de despeje de grandes fragmentos de roca que circundaban los bajorrelieves - los cuales representaban un riesgo en caso de aluviones- Ilevaron al descubrimiento de un relieve no registrado, el cual se encontraba fragmentado en 11 partes. Al final de los procesos de levantamiento, armado, montaje y restauración, ${ }^{4}$ se hizo evidente un diseño zoomorfo que sirvió de inspiración para su nombre: Tríada de los felinos (González Correa, Córdova Tello y Buitrago Sandoval 2011) (Figura 6).

En esta temporada también se realizó la intervención del Monumento 2 , que consistió en construir una cubierta de protección y unas escalinatas para acceder tanto a él como a un mirador frontal, que incluye un área de reposo para los visitantes en la obligada subida hacia los de-

\footnotetext{
${ }^{4}$ Para el montaje, la roca se trasladó y ancló al centro dentro de una caja de concreto, aislada de éste por una cama de arena. En la parte posterior se colocaron tres tornillos acerados de alta resistencia para proporcionar apoyo al monumento. Los fragmentos se unieron con resina epóxica araldita: el elemento se protegió con una techumbre (CNCPC-INAH y Centro INAH-Morelos 2011).
}

más monumentos. En concordancia con estas acciones de optimización de uso público y de conservación del entorno natural, se procedió a la plantación de 30 árboles de la región seleccionados por una bióloga del Centro INAH-Morelos, los cuales se dispusieron como áreas de sombra para la visita. Sobre el camino se sembró pasto, con lo que se logró un cambio significativo en el entorno de los senderos que hoy aglutina monumentos con representaciones de felinos.

Tercera temporada (septiembreoctubre de 2012)

En esta temporada los trabajos se enfocaron principalmente en la conservación preventiva, aunque también sobresalieron las acciones dirigidas a la adecuación y mejoramiento de las áreas que rodean los monumentos. Por lo tanto, se continuó con el mantenimiento y el monitoreo de las techumbres elaboradas en anteriores temporadas, previa comprobación de su adecuado desempeño en la protección de los relieves contra la Iluvia, el viento y los rayos directos del sol. Asimismo, en los relieves 3 o El puma y 5 o Mito de la creación del hombre se instalaron nuevas estructuras de protección optimizadas en su diseño: se disminuyó su peso y se aumentó su estabilidad, a la par que se cumplió con la finalidad de integrarlas al entorno sin interrumpir mayormente la apreciación de los monumentos. También se realizó una protección provisional para dos piezas que se habían excavado recientemente junto al relieve $\mathrm{El} \mathrm{Ca}$ zador: en este caso, la tarea se limitó a envolverlas in situ con lonas impermeables tratando de minimizar la eventual afectación por el intemperismo.

De la misma manera, para reducir la concentración y los escurrimientos de aguas de lluvia - fenómeno que acrecentaba el riesgo de impacto por deslaves de tierra y piedras al relieve Tríada de los felinos (Monumento 41) y al Felino agazapado 

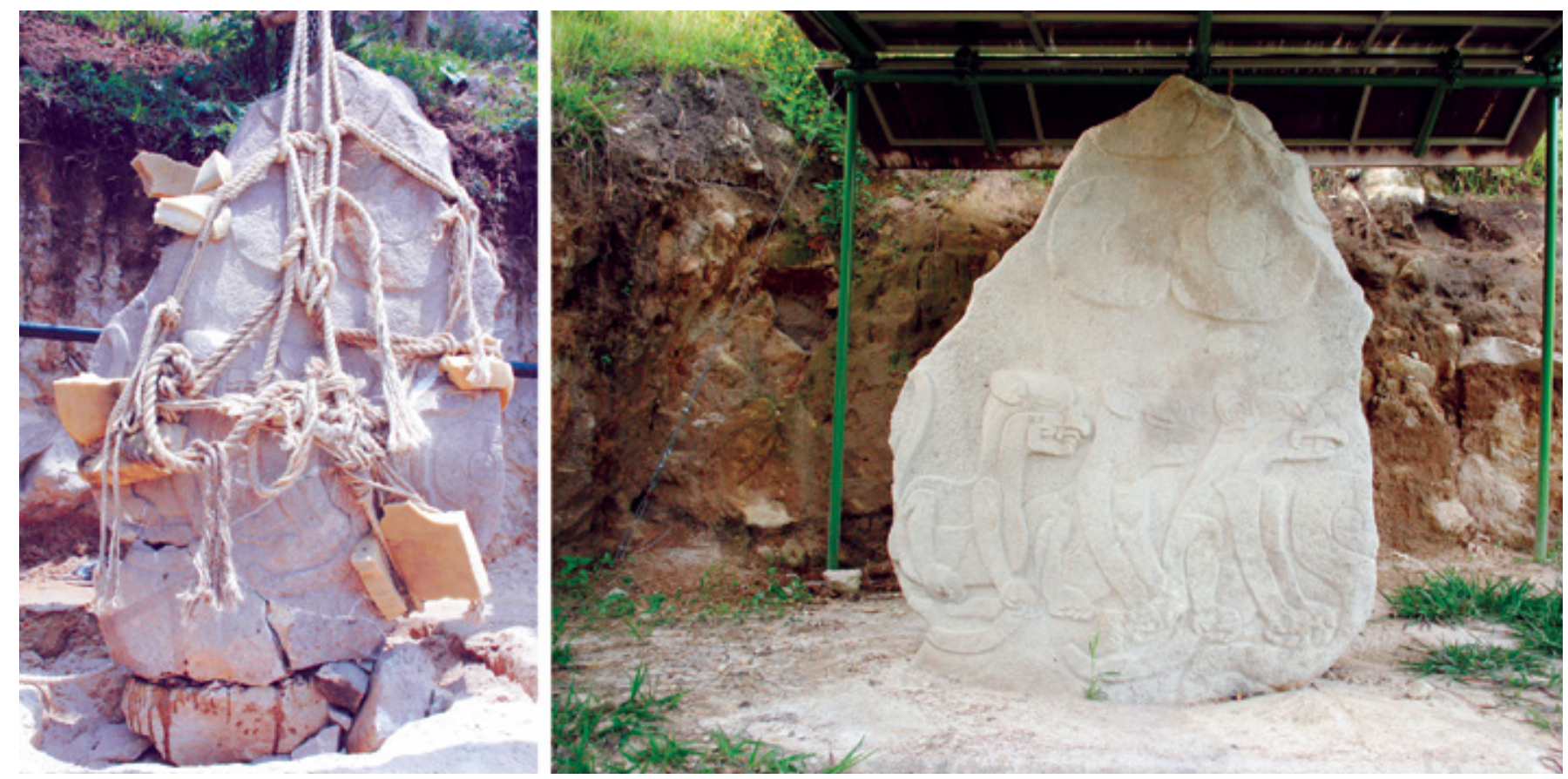

FIGURA 6. Relieve Tríada de los felinos, Zona Arqueológica de Chalcatzingo, Morelos, México, en proceso de montaje en su sitio y aspecto del monumento después de la intervención (Cortesía: Proyecto de Conservación y Restauración de los Elementos Arqueológicos de Chalcatzingo, CNCPC-INAH).

(Monumento 31) — en algunas áreas del sitio arqueológico se procedió a la construcción de canales de desagüe. Otra labor importante de conservación preventiva fue el deshierbe en áreas cercanas a los monumentos y en los senderos que conducen a ellos, ya que, como hemos mencionado, una serie de alteraciones en las rocas se asocia con el crecimiento de plantas superiores, las que producen otras afectaciones, tales como la obstrucción en la visibilidad de los monumentos, dificultades en el recorrido por la zona y la acción de animales superiores en busca de alimento o refugio.

Cuarta temporada (agostoseptiembre de 2013)

El último ciclo de trabajo de campo se centró en las siguientes labores: la intervención del Monumento 3 o El puma; el mantenimiento en áreas de circulación del recorrido de los petrograbados; la conservación preventiva y mantenimiento del Monumento 5 o Mito de la creación del hombre; y la elaboración de la cubierta del Monumento 26, que es el fragmento de la base de una estela con figuras talladas en forma de volutas.

En general, destaca el mejoramiento de los senderos de tránsito, la optimización de la lectura de los monumentos y la apreciación del sitio arqueológico por los visitantes. Hay que agregar, en lo particular, la notable intervención del Monumento 3, ya que se logró no sólo su estabilización por medio del uso de pernos sino también la virtual recuperación de la imagen representada en el relieve.

\section{Consideraciones finales}

Las acciones de investigación e intervención realizadas por el Proyecto de Conservación y Restauración de los Elementos Arqueológicos de Chalcatzingo (CNCPC-INAH) han sido benéficas en varios rubros claves: el estudio material de los relieves, una mayor profundización del diagnóstico sobre su deterioro, su consolidación, la optimización de su lectura, y el mejoramiento global de la preservación y apreciación de la Zona Arqueológica de Chalcatzingo.
A lo ya logrado se suma una reflexión sobre las tareas que habrán de emprenderse en el futuro: en materia de investigación es necesario establecer una agenda de prioridades donde converjan, por un lado, iniciativas de estudios arqueológicos específicos para entender el origen de los relieves, su iconografía y significados para la sociedad que los produjo; y por otro, la investigación de las técnicas de manufactura, análisis más específicos acerca de las rocas sobre las que se tallaron y estudios que abunden en los procesos de alteración sufridos. De especial importancia será mantener un programa de conservación preventiva con personal debidamente capacitado tanto para monitorear el sitio y vigilar que no se presenten nuevos deterioros (fisuras o pérdidas de material) —e intervenir oportunamente, en caso contrario- como para realizar verificaciones periódicas de las cubiertas de protección y retirar la vegetación que esté creciendo alrededor de los relieves. A estas actividades deberán sumarse intervenciones de conservación específicas, como ciclos de limpieza y 
estabilización, que deberán realizar conservadores profesionales.

A manera de cierre, es importante subrayar la relevancia de la conservación en específico de los monumentos de Chalcatzingo, ya que estos relieves representan la impronta del estilo olmeca del sitio y nos proporcionan información sobre rituales y ceremonias particulares de un pueblo que se asentó hace 3000 años. Se trata de "piedras que hablan", cuya supervivencia permite dialogar con sociedades de un pasado sobre el cual todavía nos falta mucho por conocer.

\section{Agradecimientos}

A las doctoras Isabel Villaseñor Alonso e Isabel Medina-González, y a la maestra Concepción Obregón Rodríguez, por sus aportes a este escrito. A los arqueólogos Mario Córdova Tello y Rogelio Rivero Chong, por su apoyo para la realización del trabajo in situ.

\section{Referencias}

CNCPC-INAH y Centro INAH Morelos

2010 Proyecto de Conservación y Restauración de los Petrograbados en la Zona Arqueológica de Chalcatzingo, Morelos. Temporada I, julio 2010, documento mecanoescrito inédito, México, Archivo de la CNCPC-INAH/Archivo del Centro INAH-Morelos.

2011 Proyecto de Conservación y Restauración de los Petrograbados en la Zona Arqueológica de Chalcatzingo, Morelos. Temporada II, marzoabril 2011, documento mecanoescrito inédito, México, Archivo de la CNCPC-
INAH/Archivo del Centro INAH-Morelos. 2012 Proyecto de Conservación y Restauración de los Petrograbados en la Zona Arqueológica de Chalcatzingo, Morelos. Temporada III, septiembreoctubre 2012, documento mecanoescrito inédito, México, Archivo de la CNCPC-INAH/Archivo del Centro INAHMorelos.

2013 Proyecto de Conservación y Restauración de los Petrograbados en la Zona Arqueológica de Chalcatzingo, Morelos. Temporada IV, julioagosto 2013, documento mecanoescrito inédito, México, Archivo de la CNCPC-INAH/Archivo del Centro INAHMorelos.

Córdova Tello, Mario y Carolina Meza Rodríguez 2007 "Chalcatzingo, Morelos. Un discurso sobre piedra", Arqueología Mexicana (XV) 87: 60-65.

García Murillo, Susana 1995 "Estudio de los procesos de bioalteración de la piedra en la catedral de Pamplona", tesis doctoral en química y edafología, Pamplona, Universidad de Navarra.

González Correa, Olga Lucía, Mario Córdova Tello y Gilberto Buitrago Sandoval 2011 "El Monumento 41 o Tríada de los felinos", Arqueología Mexicana, 111, documento electrónico disponible en [http://www.arqueomex. com/S2N3nChalcatzingo111.html], consultado en enero de 2015.

Grove, David C. y Jorge Angulo 1987 "The Chalcatzingo Reliefs: An Iconographic Analysis", en Grove 1987a:132-158.

Grove, David C. (ed.) 1987a Ancient Chalcatzingo, Austin, University of Texas Press.
Grove, David C.

1987b Introduction, en Grove (ed.)

1987a: 1-5.

1999 "Public Monuments and Sacred Mountains: Observations on Three Formative Period Sacred Landscapes", en David C. Grove y Rosemary A. Joyce (eds.), Social Patterns in PreClassic Mesoamerica: A Symposium at Dumbarton Oaks, 9 y 10 de octubre de 1993, Washington D. C., Dumbarton Oaks Research Library and Collection, 255-300.

2008 "Chalcatzingo: A Brief Introduction", The PARI Journal 9 (1): 1-7.

2010 [2008] Chalcatzingo: breve introducción, documento electrónico disponible en [http://www.mesoweb. com/pari/publications/journal/901/ Chalcatzingo.pdf], consultado en febrero de 2014, 1-8.

Medina-González, Isabel y Renata Schneider

1993 “Los líquenes y su incidencia en los materiales porosos de construcción", Imprimatura, Revista de Restauración, VII: 28-31.

Mori, Laura

2007 "Origen del magmatismo miocénico en el sector central de la FVTM [Faja Volcánica Transmexicana] y sus implicaciones en la evolución del sistema de subducción mexicano", tesis doctoral en ciencias de la tierra, México, Centro de Geociencias-unam.

Werre Keeman, Frits J. y Luis Enrique Ortiz-Hernández

2000 Monografía geológico-minera del estado de Morelos, México, Consejo de Recursos Minerales, Secretaría de Comercio y Fomento Industrial. 


\section{Juan Francisco Illescas Salinas}

Universidad Autónoma Metropolitana, Unidad Azcapotzalco (UAM-A), México

jfis@azc.uam.mx

Doctor en ciencias (Universidad de Cádiz [UCA], España) y licenciado en geología (Universidad de Granada [UGR], España). Desde 2008 participa en proyectos de investigación relacionados con el desarrollo de nanomateriales con aplicación en la conservación-restauración de materiales pétreos. Es autor de dos patentes de invención, consistentes en productos para protección y restauración de rocas. Sus líneas de investigación como profesor invitado del área de química aplicada, departamento de ciencias básicas en la Universidad Autónoma Metropolitana, Unidad Azcapotzalco (UAM-A), México, son la evaluación de parámetros ambientales como factores determinantes en el proceso sol-gel de materiales con aplicación en conservación-restauración de materiales pétreos monumentales y el desarrollo de nanocomposites con propiedades de biocontrol sobre rocas monumentales.

\author{
Gilberto Buitrago Sandoval \\ Restaurador independiente, Colombia \\ buitragosandoval@hotmail.com
}

Licenciado en restauración (Escuela Nacional de Conservación, Restauración y Museología [ENCRYM], Instituto Nacional de Antropología e Historia [INAH], México). Ha realizado trabajos de conservación y restauración en Colombia con el Ministerio de Cultura, el Banco de la República y otros organismos de gobierno. Formó parte del Proyecto para la Conservación de las Pinturas Murales de la Zona Arqueológica de Bonampak (Coordinación Nacional de Conservación del Patrimonio Cultural [CNCPC], INAH, México) entre 2010 y 2012; también ha realizado labores de conservación en la Zona Arqueológica de Chalcatzingo, Morelos, México. Actualmente dirige los trabajos de conservación y restauración de pintura mural en la Casa de la Independencia, futura sede del Museo de Bogotá, Colombia. 